\title{
Numerical simulation of press forming of an automotive body structural component using St13 and BH340 steel sheets
}

\author{
Y. Yu ${ }^{1}$, Z. Yang ${ }^{1}$, J. Chen ${ }^{1}$ \& D. O. Northwood ${ }^{2}$ \\ ${ }^{1}$ Key Laboratory of Advanced Structural Materials, \\ Ministry of Education, Changchun University of Technology, P.R. China \\ ${ }^{2}$ Department of Mechanical, Automotive and Materials Engineering, \\ University of Windsor, Canada
}

\begin{abstract}
Numerical simulation experiments using Dynaform were performed for the press forming of the front and rear roof beams of a medium-duty truck CA1092 produced by the First Automobile Factory of China. The production route for both beams involves blanking, press punching, trimming and true-up: pressing in the major production step. These beams were being produced using construction steel (St 13) sheets. As part of an initiative to reduce vehicle weight, and thereby improve performance, the company wished to replace the St13 steel sheets with high-strength steel (BH340) sheets. Press forming simulation results for BH340 steel using the same forming parameters (tool velocity, binder force, friction coefficient) as for St13 steel showed a serious tendency for the parts to wrinkle and crack. Modification of the forming parameters eliminated the cracking of the BH340 sheets and improved the thickness uniformity compared to beams made from the St13 steel sheets.

Keywords: front beam and rear beam of truck, Dynaform, numerical simulation, high-strength steel.
\end{abstract}

\section{Introduction}

Since the late 1970s and early 1980s, many countries in the world began using high-strength steel to produce automotive body components $[1,2]$. In recent years, high-strength steel consumption has increased year by year. Reducing 
vehicle weight has been a common pursuit of both domestic and foreign auto makers, since this cannot only reduce production costs, but can also save resources, e.g., improved fuel economy, and improved safety [1-11]. The move to reduce vehicle weight has lead to the increased use of inherently light weight materials such as aluminium alloys [1]. High-strength steel (HSS) sheets have contributed to lightening vehicles by reducing sheet thickness [1]. Higher strength levels, and thinner sections, have been obtained through the development of new generations of HSS, which are referred to as advanced HSS (AHSS) and extra- and ultra-AHSS [12, 13].

Application of numerical simulation of sheet metal forming technology can reduce the mould design cycle, cut down the cost of design, and improve both the quality of sheet metal and the production efficiency $[14,15]$. The front beam and rear beam are typical automotive press forming parts, and numerical simulation experiments of the press forming of them made by high strength steel BH340 and construction steel St13 have been investigated using Dynaform. HSS BH340, which is a bake-hardened steel, has found extensive application in street form in a number of Chinese automobiles, including Dongfeng, Wuling and Sharly [16]. By means of modelling, adjusting the parameters of press forming technology, and comparing the results for BH340 and St13, the impact of the main forming parameters such as tool velocity, bind hold force and friction coefficient were obtained.

The chemical compositions and the mechanical properties of the St13 and BH340 steels are shown in Tables 1 and 2, respectively.

\section{Modelling and pre-processing}

\subsection{Analysis setup}

As shown in Figure 1, the following route was adopted: Tool, Analysis setup, the length unit is $\mathrm{mm}$; the draw type is single action; the contact direction is form one way $\mathrm{S}$ to $\mathrm{S}$; the stroke direction is $\mathrm{Z}$; and contact gap is 1.00 .

Table 1: $\quad$ Chemical composition of BH340 and St13 steels.

\begin{tabular}{cccccccccc}
\hline $\begin{array}{c}\text { Element } \\
\text { Wt. \% }\end{array}$ & C & Si & Mn & P & S & Al & N & Ti & Nb \\
\hline BH340 & $\leq 0.02$ & $\leq 0.10$ & $\leq 0.40$ & 0.083 & $\leq 0.030$ & $\geq 0.02$ & 0.003 & $/$ & $/$ \\
\hline St13 & $\leq 0.08$ & $/$ & $\leq 0.45$ & $\leq 0.03$ & $\leq 0.015$ & $\geq 0.035$ & $/$ & $/$ & $/$ \\
\hline
\end{tabular}

Table 2: $\quad$ The mechanical properties of BH340 and St13 steels.

\begin{tabular}{ccccccc}
\hline Steel & $\sigma \mathrm{s}(\mathrm{MPa})$ & $\sigma \mathrm{b}(\mathrm{MPa})$ & $\delta(\%)$ & $\mathrm{r}$ & $\mathrm{n}$ & $\mathrm{BH}$ \\
\hline $\mathrm{BH} 340$ & $\geq 185$ & $\geq 340$ & $\geq 34(\mathrm{Lo}=80 \mathrm{~mm})$ & $\geq 1.6$ & $\geq 0.20$ & $\geq 30$ \\
\hline St13 & $\leq 240$ & $270 \sim 370$ & $\geq 34(\mathrm{Lo}=80 \mathrm{~mm})$ & $/$ & $/$ & $/$ \\
\hline
\end{tabular}




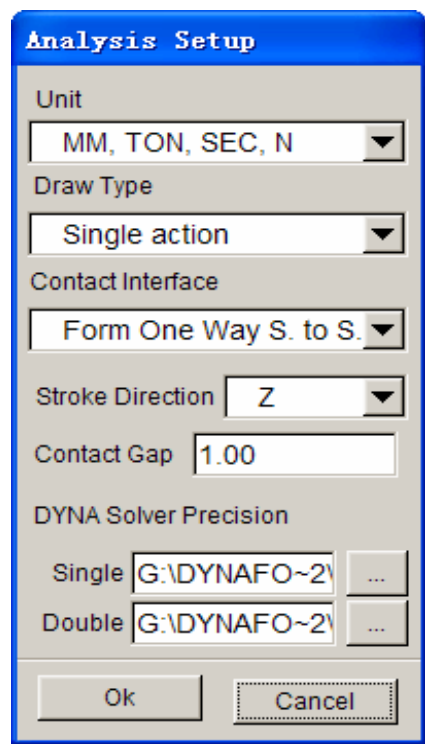

Figure 1: Analysis setup.
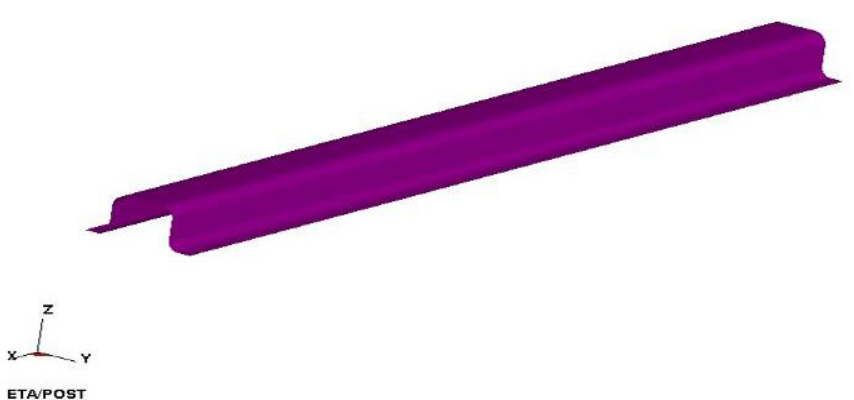

Figure 2: Three dimensional model of front and rear beam.

\subsection{Modelling}

As shown in Figure 2, the beam model was built with Dynaform. The thickness of the part was $0.9 \mathrm{~mm}$ made from BH340 steel and $1.0 \mathrm{~mm}$ made from St13 steel. The production route was blanking, press, punching, trimming and true-up, and press is the major step.

\subsection{Tool meshing}

Tool mesh was selected and the part was meshed with the default parameters: Figure 3. 
Figure 3: $\quad$ Tool mesh of the beam.

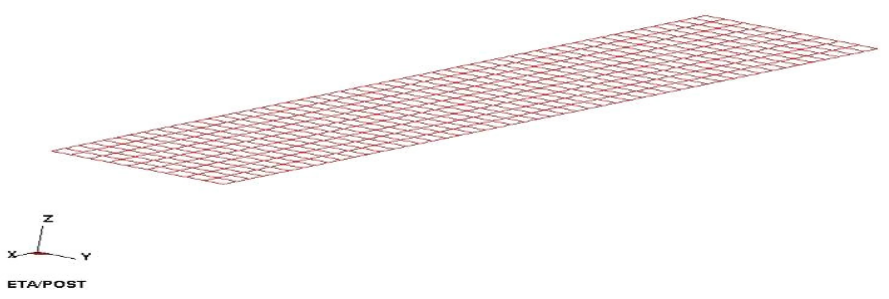

Figure 4: $\quad$ Blank mesh of the beam.

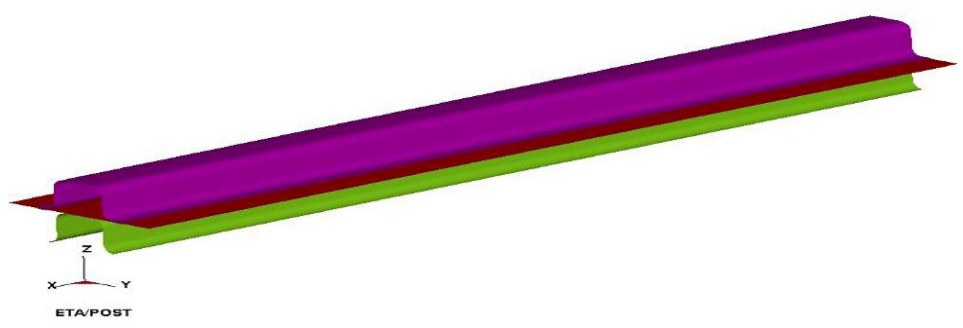

Figure 5: Tools setup.

\subsection{Blank meshing}

After filling the hole, a model boundary check, a normal consistency check and a series of mesh check and repair procedures, the blank is generated according to the outline of the result of blank size estimate and meshed with a quadrilateral grid, divided into 5152 elements: Figure 4.

\subsection{Tools setup}

The tools are defined in the toolbar. As shown in Figure 5, these are die, blank, binder and punch from above to below. 


\section{Results and discussion}

\subsection{Press forming simulation results for construction steel sheet St13}

In the press forming simulation of the vehicle beam, and in order to enhance the computational efficiency, the conditions are approximated with a Hill yield criterion for anisotropic materials model being adopted for the blank material, while a rigid material model was used for the mould materials.

Based on the results of actual forming experiments, a tool velocity of $8000 \mathrm{~mm} / \mathrm{s}$ and a binder force of $800 \mathrm{KN}$ were used for the simulation. The results for a beam made of construction steel sheet St13 of thickness $1.0 \mathrm{~mm}$, are shown in Figures 6 and 7.

From Figure 6 of FLD for press forming of the beam made of St13, we can see that there is no wrinkling or cracking in the part.

From Figure 7 for the thickness contours we can see that the minimum thickness is $0.933317 \mathrm{~mm}$, a $6.67 \%$ decrease from the original blank thickness. The maximum thickness is $1.000894 \mathrm{~mm}$, a $0.08 \%$ increase from the original thickness. These changes are both less than general quality criteria of a decrease of $30 \%$ and an increase of $10 \%$.

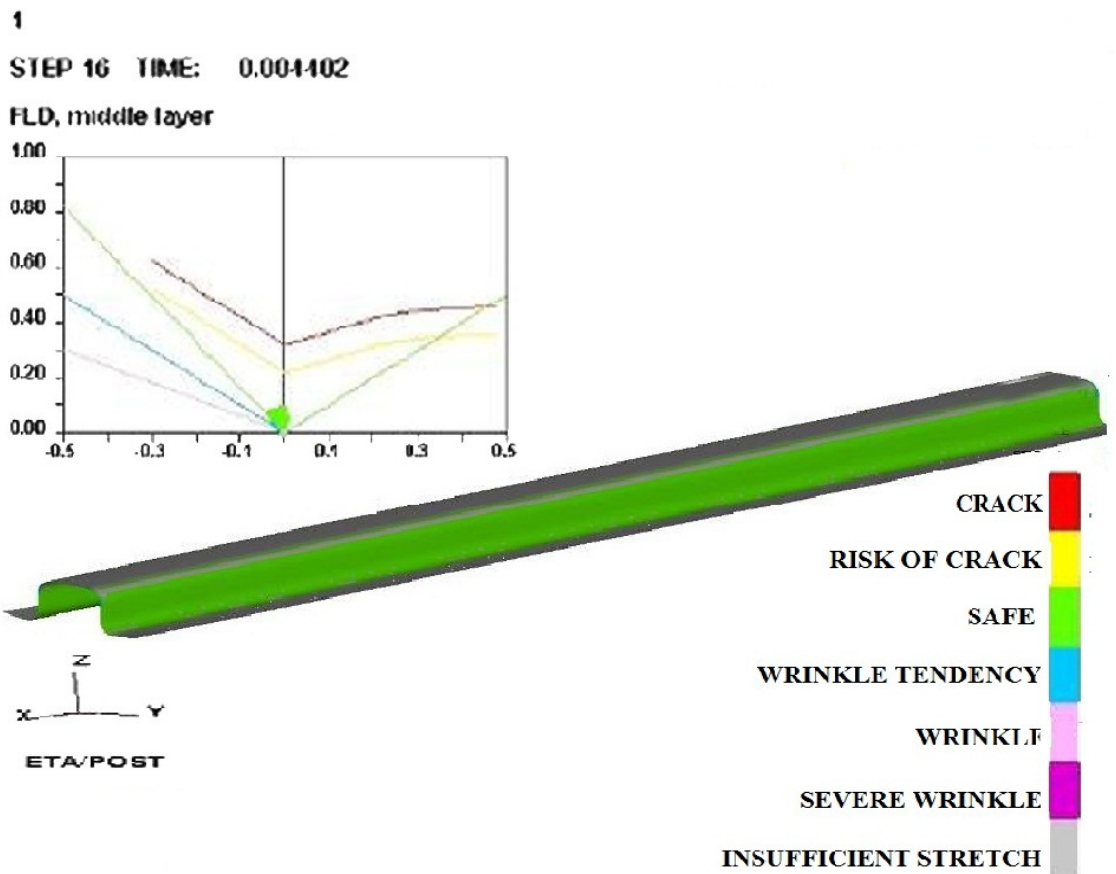

Figure 6: $\quad$ FLD for press forming of a beam made from St13 steel sheet. 


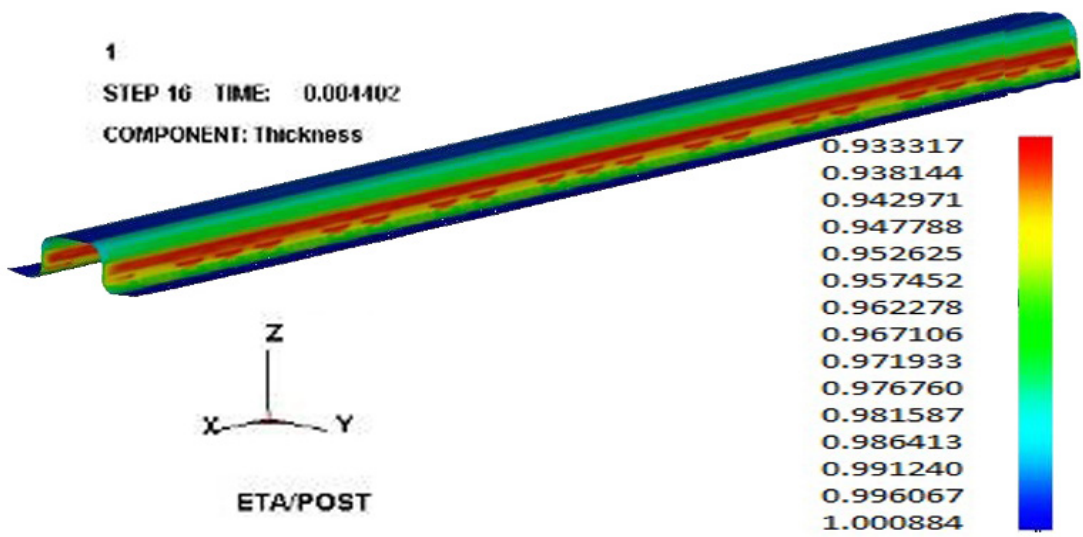

Figure 7: Thickness contour for press forming of the beam made from St13 steel sheet.

\subsection{Press forming simulation results for $\mathrm{BH} 340$ steel with the same forming parameters as for St13 steel}

The press forming simulation results of a beam made of high-strength steel sheet BH340 of thickness $0.9 \mathrm{~mm}$ are shown in Figure 8, when the forming parameters were the same as for St13 steel.

It can be seen that there is a serious tendency to wrinkling and cracking in the part, and that it cracks in the bottom corner. Because the shape transition of the part is relatively sharp, and after undergoing bending or unbending deformation, the generated stress tends to be more than the tensile limit of the sheet metal and causes it to crack. At the same time, the binder force is too high, limiting the inflow of the material, and exacerbating the tendency to crack. In order to solve the defect problem, we should reduce the resistance when the sheet metal flows into the die. Reducing the thickness of the sheet metal, increasing the lower die fillet radius, decreasing the die friction coefficient and the blank holder force are all effective measures.

\subsection{Press forming simulation results of BH340 with the forming parameters modified}

In the deep pressing process, higher tensile stresses can help avoid wrinkling and the blank holder force is the main factor controlling the material flow. Excess blank holder force leads to the risk of cracking, while insufficient blank holder force leads to faster sheet metal flow and wrinkling occurs. Therefore, the blank holder force in the press forming process should be in a range where neither wrinkling nor cracking occurs. 
The modified forming parameters, again chosen on the basis of the results of actual forming experiments, were tool velocity $5000 \mathrm{~mm} / \mathrm{s}$, binder force $600 \mathrm{KN}$. The results are shown in Figure 9 and Figure 10. Compared with the results with no modification of the forming parameters (Figure 8), it can be seen that cracking is eliminated.

It can be seen from Figure 10 that the minimum thickness of the beam made from $\mathrm{BH} 340$ steel of thickness $0.9 \mathrm{~mm}$ is $0.876066 \mathrm{~mm}$, a $2.66 \%$ reduction in thickness. This compares with a reduction of $6.67 \%$ for the St13 steel beam. Thus, the thickness uniformity of the product has been improved by replacing St13 steel with BH340 steel.

The optimal steel press forming parameters for the fabrication of beams with the different two steels are shown in Table 3.

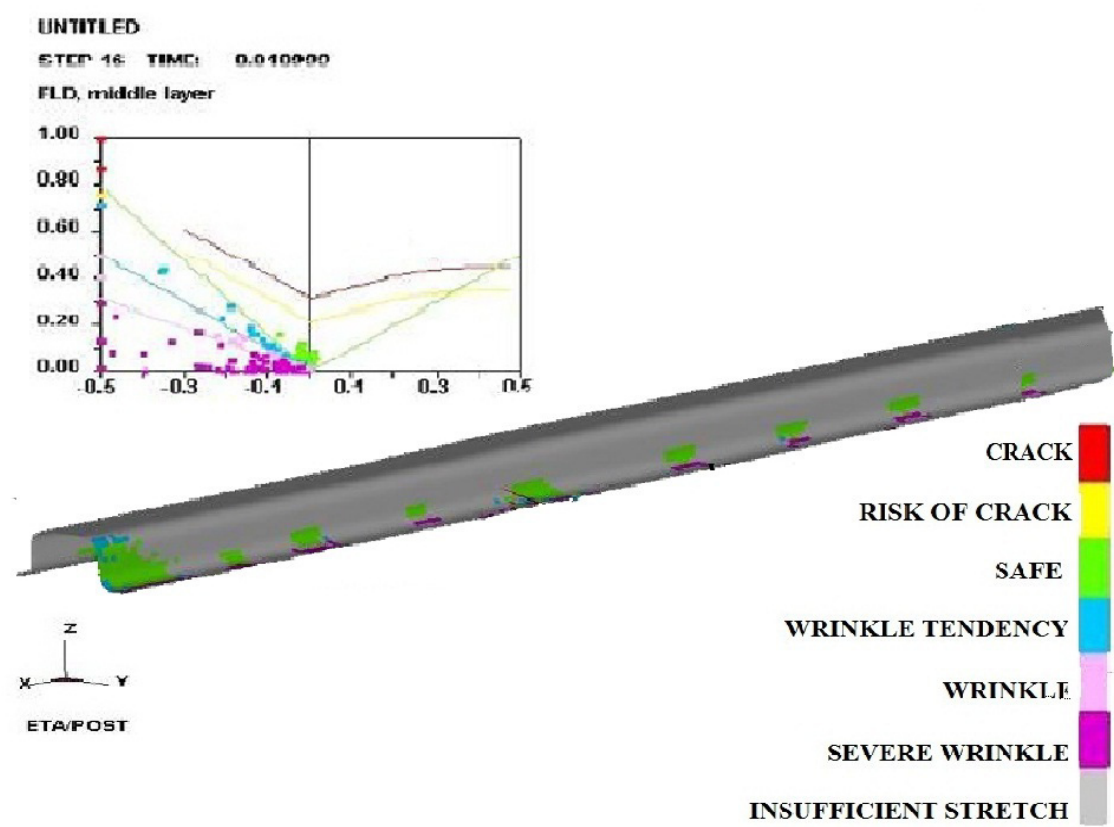

Figure 8: $\quad$ FLD for forming of a beam made from BH340 steel using the same forming parameters as for St13 steel.

Table 3: Optimal simulation forming parameters for beams fabricated with different steels.

\begin{tabular}{cccc}
\hline Steel & Thickness $/ \mathrm{mm}$ & Tool velocity $/ \mathrm{mm} \bullet \mathrm{s}^{-1}$ & Binder force $/ \mathrm{N}$ \\
\hline St13 & 1.0 & 8000 & 800,000 \\
\hline BH340 & 0.9 & 5000 & 600,000 \\
\hline
\end{tabular}


1

SIEP 16 TIME: $0.00+402$

FLo. Maddle Iayer
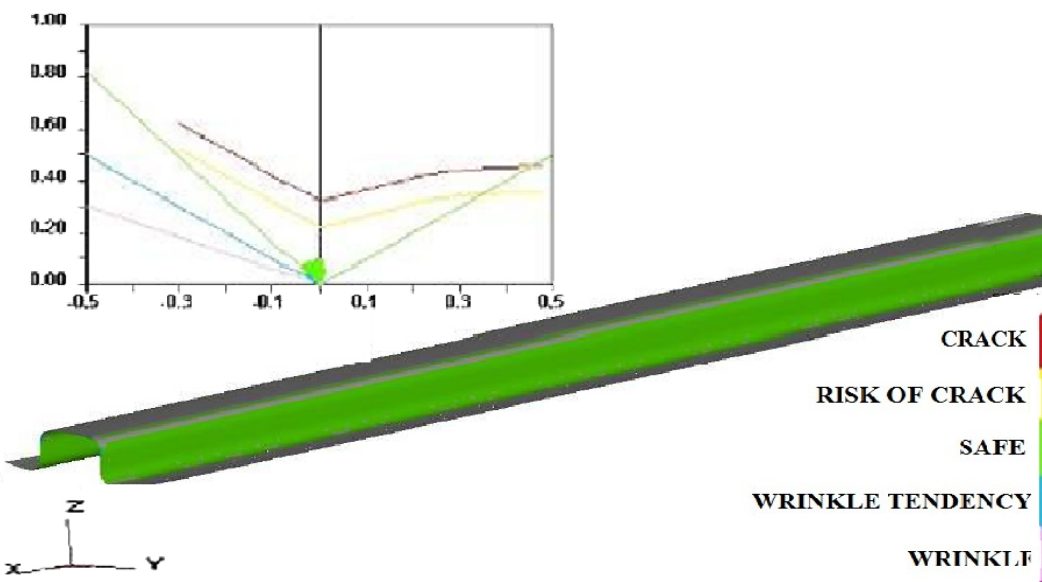

WRINKLE TENDENCY

ETAPOST

WRINKI.T

SEVERE WRINKLE

INSUFFICIENT STRETCH

Figure 9: $\quad$ FLD for proper press forming of the beam made of BH340.

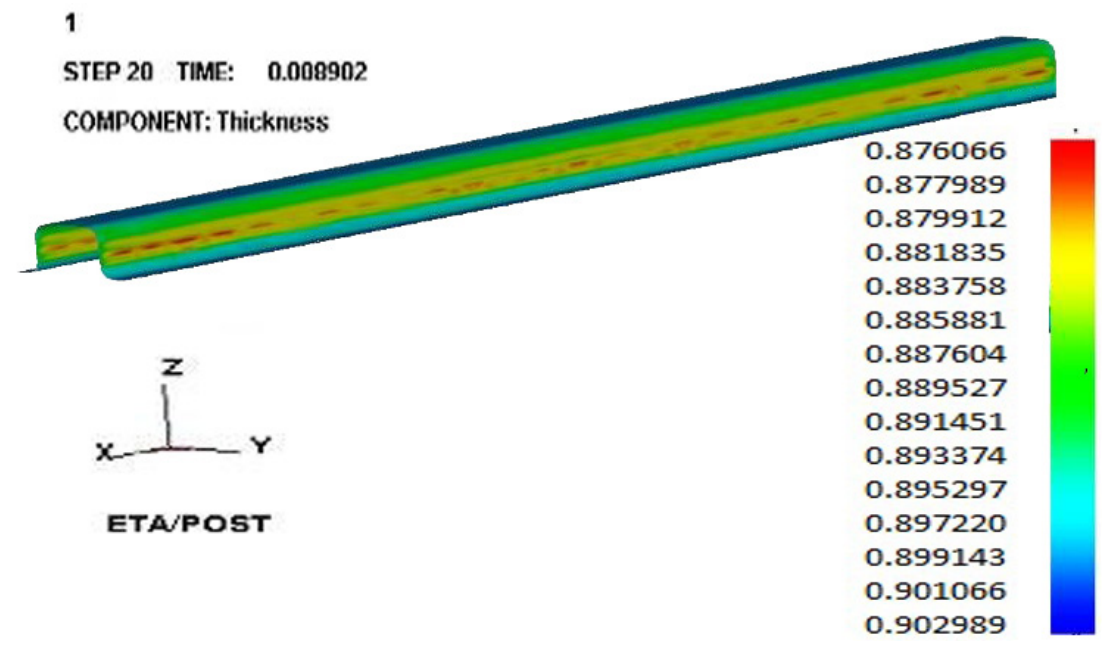

Figure 10: Thickness contour for proper press forming of the beam made of BH340. 


\section{Conclusions}

This study has shown that:

1. BH340 steel shows good forming performance after modifying the press forming parameters such as tool velocity, bind hold force and friction coefficient from those used for St13 steel.

2. The thickness uniformity is improved compared to St13 steel.

3. The optimal parameters for the press forming of the beams are tool velocity $5000 \mathrm{~mm} / \mathrm{s}$, binder force $600 \mathrm{KN}$ for the BH340 steel.

On the basis of the results of this study, BH340 steel has been utilized in the commercial production of the front and rear roof beams for the CA1092 medium-duty truck.

\section{References}

[1] Shibata, K. Future vehicles and materials technologies. Automotive Engineering: Lightweight, Functional and Novel Materials, eds B. Cantor, P. Grant \& C. Johnson, CRC Press: Boca Raton, FL, USA, pp. 3-17, 2008.

[2] Charles, J.A. \& Crane, F.A.A., Materials for automobile structures (Chapter 18). Selection and Use of Engineering Materials: $2^{\text {nd }}$ Edition, Butterworths: Tiptree, UK, pp. 298-308, 1989.

[3] Kwon, O. \& Baik, S.C., Manufacture and application of advanced high strength steel sheets for auto parts manufacture. http://www.metal.citic.com /iwcm/UserFiles/img/cd/2005-HSLA-NB/HSLA-015.pdf.

[4] Deng, Z. \& Wang, X., Development of sheet metal for automobile industry and its measures. Kang T'ieh / Iron and Steel (Peking), 28(6), pp. 73-78, 1993 (In Chinese).

[5] Li, Y., Lin, Z., Jiang, A. \& Chen, G., Use of high strength steel sheet for light weight and crashworthy car body. Materials \& Design, 24, pp. 177182, 2003.

[6] Hayashi, H., \& Nakagawa, T., Recent trends in sheet metals and their formability in manufacturing automotive panels. Journal of Materials Processing Technology, 46 (3-4), pp. 455-487, 1994.

[7] Kang, Yongling Advances in the Development of Automobile Steel Sheets Abroad, Bao Steel Technology, pp. 3-5, Vol 1. China Academic Journal Electronic Publishing House, 1999 (In Chinese, http://www.cnki.net).

[8] Yoshida, K., Trends in forming technology for car body in Japan. Advanced Technology of Plasticity, (1):35-37, 1999.

[9] Wang X., Tang H., Li X. \&Kang Y., Current Situation and Development of Automotive steel sheet, Materials, Process and Equipment, 4, pp. 12-14, 1993 (In Chinese).

[10] Li G. Development and production of automotive sheet, China Metallurgy, 1, pp.15-18, 2002 (In Chinese, http://www.cnki.net).

[11] Manabu T., Metallurgical aspect of sheet steels of automobiles (1) Materials Science \& Technology, 74(5), 459-466, 2004. 
62 High Performance Structures and Materials V

[12] American Iron and Steel Institute, Formability Characterization of a New Generation of High-Strength Steels, AISI/DOE Technology Roadmap Program Final Report, 12pp, March 2003. www.autosteel.org.

[13] Kwon, O., Lee, K., Kim, G. \& Chin, K.-G., New trends in advanced high strength steel developments for automotive application. Materials Science Forum, 638-642, pp. 136-141, 2010.

[14] Peng Y., Metal forming simulation technology, University of Shanghai Communication, pp35-45, 1999 (In Chinese).

[15] Zhang Xiao-jing, Zhou Xian-bin. Numerical Simulation Technology on Multi-operation Sheet Forming Process, Journal of Plasticity Engineering, 8(1), pp. 25-30, 2001(In Chinese).

[16] Baosteel, http://www.baosteel.com/plc/english/e05development/e050103. htm 